\title{
Some Further Results on Fixed-Time Synchronization of Neural Networks with Stochastic Perturbations
}

\section{Aminamuhan Abudireman, Mairemunisa Abudusaimaiti, Wanjuan Sun, Jiangyuan Zhao, Yuanshuang Zhang, Abdujelil Abdurahman*}

College of Mathematics and System Sciences, Xinjiang University, Urumqi, China

Email: *abjil1@163.com, *abdujelil@xju.edu.cn

How to cite this paper: Abudireman, A., Abudusaimaiti, M., Sun, W.J., Zhao, J.Y., Zhang, Y.S. and Abdurahman, A. (2022) Some Further Results on Fixed-Time Synchronization of Neural Networks with Stochastic Perturbations. Journal of Applied Mathematics and Physics, 10, 200-218. https://doi.org/10.4236/jamp.2022.101015

Received: December 8, 2021

Accepted: January 23, 2022

Published: January 26, 2022

Copyright $\odot 2022$ by author(s) and Scientific Research Publishing Inc. This work is licensed under the Creative Commons Attribution International License (CC BY 4.0).

http://creativecommons.org/licenses/by/4.0/

\begin{abstract}
In this paper, fixed-time (FXT) synchronization issue of a type of neural networks (NNs) with stochastic perturbations is considered. First, we obtained some novel sufficient criteria to guarantee the FXT synchronization of considered networks via introducing two types of controllers and employing some inequality techniques. Lastly, our theoretical results are verified via giving two numerical examples with their Matlab simulations.
\end{abstract}

\section{Keywords}

Fixed-Time Stability, Stochastic Perturbation, Synchronization, Neural Network

\section{Introduction}

In the last decades, the various types of neural networks (NNs) including Hopfield NNs, cellular NNs, convolution NNs, Cohen-Grossberg NNs, BAM NNs and so on [1] [2] [3], have been introduced and broadly investigated due to their important applications in great number of fields ranging from speech recognition [4] to image encryption [5], from secure communication [6] to robotic manipulators [7], etc. As pointed out in [8], stability of NNs is prerequisite in some applications. As a result, the stability analysis of NNs has been investigated extensively by many scholars [2] [3] [8] [9] [10] [11] [12]. Duo to the security reasons or just to improve system performance in many practical applications, it is desirable that the systems solution trajectories converge to equilibrium as fast as possible [13]. Compared to the classical Lyapunov stability such as asymptotical stability or exponential stability, finite-time (FNT) stability allows the solution of 
an asymptotic system approaches to the equilibrium state after a some bounded time $T\left(x_{0}\right)$ and stays at equilibrium state any time longer than $T\left(x_{0}\right)$, where $T\left(x_{0}\right)$ is called the settling time. Thus, FNT stability and stabilization of NNs have been investigated widely in the past two decades [10] [11] [12] [13] [14].

One of the important tasks in FNT stability is to estimate the settling time (ST) $T\left(x_{0}\right)$, and it is desirable to obtain smaller upper-bound of $T\left(x_{0}\right)$. However, in some cases, it is inconvenient to accurately estimate it due to its heavily dependence to the initial values of the system. So it is to better obtain FNT stability with a ST irreverent to initial conditions of the system. This issue was firstly studied by Polyakov [15] via defining a so-called fixed-time (FXT) stability which its ST is independent to systems initial conditions. Presently, FXT stability receives a hot research attention from many scholars since it has awesome applications in multi-agent systems [16], power systems [17], complex networks [18] and so on.

In addition, the synchronization of chaotic nonlinear system has received great attention in the past thirty years due to the fact that synchronization is unique in nature and plays a crucial role in many fields including biology, climatology and sociology, etc. [19]. As mentioned in [9], synchronization in neuronal systems can produce a lot of physiological mechanisms of brain functions such as attention, learning, memory formation and so on. Thus, to understand these brain functions deeply, it is an important task to study synchronization behaviors of NNs. For this reason, considerable efforts have been devoted to study the synchronization of NNs [5] [6] [20]-[25]. Especially, due to the advantage of faster convergence rate, better robustness and disturbance rejection properties, FNT and FXT synchronization of various NNs have studied recently. For example, in [9], the authors investigated the FXT synchronization of coupled discontinuous NNs by introducing new FXT stability results for dynamical systems. In [11], the authors considered FNT stabilization issue of a class of delayed memristive NNs with discontinuous right-hand side by designing two types of discontinuous controllers. In [21], the authors concerned the FXT synchronization of a class of memristor-based NNs with impulsive effects. In [26], the authors studied the FNT synchronization of a type of complex-valued NNs with distributed delays. In [27], the authors studied the FXT synchronization problem of a type of quaternion-valued NN with time delays.

However, it is worthy to note that most of the above mentioned results have only considered cases without stochastic perturbations. As depicted in [28], noises are frequently encountered in both nature and man-made systems. For instance, synaptic transmission in the real nervous systems can be seen as a noisy process which is caused by random fluctuations due to the release of neurotransmitters and other probabilistic effects. Besides, for many natural renewable energy resources such as wind or solar radiation, their availability is somewhat subject to stochastic fluctuations [19]. Therefore, recently many scholars paid their attention to study the synchronization of NNs with stochastic perturba- 
tions, and till now there are many excellent results on the complete synchronization, lag synchronization, projective synchronization and FNT synchronization of stochastic NNs with or without time-delays. But, up to now, there are very few results on the FXT synchronization of stochastic NNs.

Inspired by what mentioned above, in this paper, we considered the FXT synchronization of a type of NNs with stochastic perturbations via using some improved FXT stability results. The main contributions of this work can be stated as follows. 1) Some earlier results on FXT stability of deterministic nonlinear systems are extended to the stochastic nonlinear systems. 2) Some novel sufficient conditions guaranteeing the FXT synchronization of considered stochastic NNs are derived via introducing two types of controllers and employing some inequality techniques. Lastly, two numerical examples with simulations are provided to show the feasibility of our theoretical results.

The rest of the article is structured as follows. In Section 2, some basic assumptions together with our new FXT stability lemma are proposed. Our main results on FXT synchronization in probability are given in Section 3. Two numerical examples are provided in Section 4 and our main conclusions are given in Section 5 .

Notations: The notations are quite standard. Throughout this paper, $R^{+}$and $R^{n}$ denotes the set of nonnegative real numbers and the $n$-dimensional Euclidean space, respectively. The superscript $\mathrm{T}$ represents the matrix or vector transposition. The $n \times n$ identity matrix is denoted as $I_{n} \cdot\|\cdot\|$ is the Euclidean norm in $R^{n} . N_{+}$denotes the set of positive integers. Moreover, $\left(\Omega, F, P,\left\{F_{t}\right\}_{t} \geq 0\right)$ stands for the complete probability space, where $\left\{F_{t}\right\}_{t} \geq 0$ represents the filtration satisfying the usual conditions [29]. Notation $E\{\cdot\}$ denotes for the operator of mathematical expectation corresponds to the given probability measure $P . \quad \lambda_{\max }(\cdot)$ represents the maximum eigenvalue of a real symmetric matrix.

\section{Problem Formulation and Preliminaries}

Consider a class of $\mathrm{n}$-dimensional stochastic NNs depicted by the following equation

$$
\mathrm{d} x(t)=[-D x(t)+A h(x(t))+J] \mathrm{d} t+\sigma(t, x(t)) \mathrm{d} \omega(t),
$$

where $x(t)=\left(x\left(t_{1}\right), x\left(t_{2}\right), \cdots, x\left(t_{n}\right)\right)^{\mathrm{T}}$ represents the state vector of the network at time $t, h(x(t))=\left(h_{1}\left(x_{1}(t)\right), h_{2}\left(x_{2}(t)\right), \cdots, h_{n}\left(x_{n}(t)\right)\right)^{\mathrm{T}}$ stands for the activation function of neurons; $D=\operatorname{diag}\left(d_{1}, d_{2}, \cdots, d_{n}\right)$ with $d_{i}>0$ represents to the self-feedback connection weight matrix; $A=\left(a_{i j}\right)_{n \times n_{n}}$ represents the connection weight matrix between neurons; $J=\left(J_{1}, J_{2}, \cdots, J_{n}\right)^{\mathrm{T}} \in R^{n}$ denotes neuron input vector; $\omega(t)=\left(\omega_{1}(t), \cdots, \omega_{n}(t)\right)^{\mathrm{T}}$ is an $n$-dimensional Brown motion defined on a complete probability space $(\Omega, F, P)$ with a natural filtration $\left\{F_{t}\right\}_{t} \geq 0$ generated by $\omega(s): 0 \leq s \leq t$. For more explanations about stochastic process, please see the works [19] [22] [29]. 
The corresponding response system of the drive system (1) is given by

$$
\mathrm{d} y(t)=[-D y(t)+A h(y(t))+J+u(t)] \mathrm{d} t+\sigma(t, y(t)) \mathrm{d} \omega(t),
$$

where $y(t)=\left(y\left(t_{1}\right), y\left(t_{2}\right), \cdots, y\left(t_{n}\right)\right)^{\mathrm{T}}$ denotes the state vector of the response system, $u(t)=\left(u_{1}(t), u_{2}(t), \cdots, u_{n}(t)\right)^{\mathrm{T}}$ is the feedback controller to be introduced. Other parameters $A, D, J, \sigma(\cdot)$ and $\omega(\cdot)$ are the same as defined in system (1).

In the paper, we assume that the following assumptions are satisfied for the system (1).

Assumption 1 The neuron activation functions $h_{i}$ in system (1) satisfy the Lipschitz condition. That is, for each $i$ there exists a positive constant $L_{i}$ such that

$$
0 \leq \frac{h_{i}(u)-h_{i}(v)}{u-v} \leq L_{i}, \quad \forall u, v \in R^{n},
$$

where $L_{i}>0$.

Assumption 2 For $\sigma(t, e(t))$, there exists a matrix $G \geq 0$ with appropriate dimensions such that the following inequality holds true

$$
\operatorname{trace}\left[\sigma^{\mathrm{T}}(t, e(t)) \sigma(t, e(t))\right] \leq e^{\mathrm{T}}(t) G e(t), \quad \forall(t, e) \in R^{+} \times R^{n} .
$$

Now let $e(t)=y(t)-x(t)$ be the synchronization error between drive-response systems (1) and (2), then the error dynamical system can be derived as follows:

$$
\mathrm{d} e(t)=[-D e(t)+A g(e(t))+u(t)] \mathrm{d} t+\sigma(t, e(t)) \mathrm{d} \omega(t),
$$

where $g(e(t))=h(y(t))-h(x(t))$ and $\sigma(t, e(t))=\sigma(t, y(t))-\sigma(t, x(t))$.

Furthermore, to obtain our main results, we give some related properties of stochastic perturbation, which can be found in [29].

Denote by $C^{2,1}\left(R^{n} \times R^{+} ; R^{+}\right)$the set of all nonnegative functions $V(t, z)$ on $R^{n} \times R^{+}$which its first order derivative exist for $t$ and second order derivative exist for $z$. For each $V \in C^{2,1}\left(R^{n} \times R^{+} ; R^{+}\right)$, we define an operator $£ V$ from $R^{n} \times R^{+}$to $R$ given as

$$
£ V(z, t)=V_{t}(z, t)+V_{z}(z, t) f(t, z(t))+\frac{1}{2} \operatorname{trace}\left[\sigma^{\mathrm{T}}(t, z(t)) V_{z z} \sigma(t, z(t))\right],
$$

where $V_{t}(z, t)=\frac{\partial V(z, t)}{\partial t}, V_{z}(z, t)=\left(\frac{\partial V(z, t)}{\partial z_{1}}, \cdots, \frac{\partial V(z, t)}{\partial z_{n}}\right)$,

$V_{x x}=\left(\frac{\partial^{2} V(z, t)}{\partial z_{i} \partial z_{j}}\right)_{n \times n}$.

Now consider the following general stochastic nonlinear system:

$$
\mathrm{d} z(t)=f(z(t)) \mathrm{d} t+g(z(t)) \mathrm{d} \omega(t), \quad z(0)=z_{0},
$$

where $z(t) \in R^{n}$ is state vector of system, $\omega(t)=\left(\omega_{1}(t), \cdots, \omega_{n}(t)\right)^{\mathrm{T}}$ is an $n$-dimensional Brown motion defined on a complete probability space $(\Omega, F, P) . f(\cdot): R^{n} \rightarrow R^{n}$ and $g(\cdot): R^{n} \rightarrow R^{n \times m}$ are nonlinear vector-valued continuous functions and they satisfy the condition $f(0)=g(0)=0$. 
For convenience, we denote by $z(t)=z\left(t, z_{0}\right)$ the solution of stochastic nonlinear system (4) satisfy the initial value $z(0)=z_{0}$. Also, in order to get our main results in this part, we state here some needed definitions and lemmas as follows.

Definition 1 (FXT stable in probability [30]). The zero solution of stochastic system (4) is called to be FNT stable in probability, if the following conditions hold true.

1) FNT attractiveness in probability. That is, for any initial conditions $z_{0}(\neq 0) \in R^{n}$, the equation $\operatorname{Pro}\left\{T\left(z_{0}, \omega\right)<\infty\right\}=1$ is satisfied, where $T\left(z_{0}, \omega\right)$ is ST function defined as $T\left(z_{0}, \omega\right) \triangleq \inf \left\{T>0 \mid z\left(t, z_{0}\right)=0, t \geq T\right\}$;

2) Stability in probability: For every pair of scalers $0<\mu<1$ and $r>0$, there exists a positive constant $\Delta=\Delta(\mu, r)>0$ such that $\operatorname{Pro}\left\{\left|z\left(t, z_{0}\right)\right| \leq \Delta\right\} \geq 1-\mu$.

Definition 2 (FXT stable in probability [30]). The zero solution $z=0$ of system (4) is said to be globally FXT stable in probability, if the following statements are satisfied for all the initial states $z_{0} \in R^{n}$.

1) The zero solution $z=0$ is globally stochastically FNT stable in probability.

2) Mathematical expectation of ST function $T\left(z_{0}, \omega\right)$ is independent on the initial state $z_{0}$ of (4) and its upper bound is bounded by a positive constant $T_{\max }$. That is, $E\left(T\left(z_{0}, \omega\right)\right) \leq T_{\max }$ for all $z_{0} \in R^{n}$.

Now, we introduce the following lemmas about FXT stability.

Lemma 1 [30]. Assume that $V(z): R^{n} \rightarrow R^{+}$is a positive definite Lyapunov function, $r(v)$ is a continuous function and it belongs to set $V_{M}^{0}$, where $V_{M}^{0}$ denotes set of the bounded functions which is defined be

$$
V_{M}^{0}=\left\{r(z) \mid \int_{0}^{\infty} \frac{1}{r(z)} \mathrm{d} z \leq M \text { and } r^{\prime}(z) \geq 0\right\},
$$

where $M$ is positive constant. If the following inequality is satisfied for all $z(t)=z\left(t, z_{0}\right), z_{0} \in R^{n}$

$$
£ V(z) \leq-r(V(z)),
$$

then the zero solution $z=0$ of system (4) is globally stochastically FXT stable in probability and the its ST function $T\left(z_{0}, \omega\right)$ can be estimated as $E\left[T\left(z_{0}, \omega\right)\right] \leq M$.

Lemma 2 [20]. For system (4), if there exists a positive definite function $V(z): R^{n} \rightarrow R^{+}$and positive numbers $a, b, c, \theta, \delta$ satisfying $a, b, c>0$, $0<\theta<1<\delta$ such that

$$
£ V(z) \leq c V(z)-a V^{\theta}(z)-b V^{\delta}(z), \quad \forall z(t) \in R^{n} \backslash\{0\},
$$

then the zero solution of system (4) is globally stochastically FXT stable in probability, and its ST $T\left(z_{0}, \omega\right)$ can be estimated as

$$
E\left[T\left(z_{0}, \omega\right)\right] \leq T_{\max }^{1} \triangleq \frac{1}{(1-\theta)(a-c)}+\frac{1}{(b-c)(\delta-1)} .
$$


Lemma 3 [31]. Suppose $V(\cdot): R^{n} \rightarrow R^{+} \cup\{0\}$ is a positive definite function, and it satisfies the following conditions.

1) $V(z(t))=0 \Leftrightarrow z(t)=0$;

2) For any solution $z(t)$ of system (4), following inequality hold true

$$
£ V(z(t)) \leq-c V(z(t))-a V^{\theta}(z(t))-b V^{\delta}(z(t)), \quad \forall z(t) \in R^{n} \backslash\{0\}
$$

for some $a, b>0, c>0,0<\theta<1$ and $\delta>1$. Then the zero solution of system (4) can achieve FXT stability, and its corresponding ST $T\left(z_{0}, \omega\right)$ can be estimated by

$$
E\left(T\left(z_{0}, \omega\right)\right) \leq T_{\max }^{2}=\frac{1}{c(1-\theta)} \ln \left(1+\frac{c}{a}\right)+\frac{1}{c(\delta-1)} \ln \left(1+\frac{c}{b}\right) .
$$

Lemma 4 [23]. Suppose $V(z): R^{n} \rightarrow R$ is a $C$-regular function such that

$$
£ V(z(t)) \leq-c V(z(t))-a V^{\theta}(z(t))-b V^{\delta}(z(t)), \quad z(t) \in R^{n} \backslash\{0\},
$$

where $c \in R, a>0, b>0, \delta>1$, and $0<\theta<1$, then the following results are true.

1) If $c \geq 0$, the zero solution of system (4) is FXT stable and its settling-time $T\left(z_{0}, \omega\right)$ is estimated by

$$
E\left(T\left(z_{0}, \omega\right)\right) \leq T_{\max }^{3}=\frac{\pi}{(\delta-\theta) a}\left(\frac{a}{b}\right) \csc (\varepsilon \pi),
$$

where $\varepsilon=\frac{1-\theta}{\delta-\theta}$.

2) If $0<-c<\min \{a, b\}$, the zero solution of system (4) is FXT stable and its ST $T\left(z_{0}, \omega\right)$ is estimated by

$$
\begin{aligned}
E\left(T\left(z_{0}, \omega\right)\right) \leq T_{\max }^{4}= & \frac{\pi \csc (\varepsilon \pi)}{b(\delta-\theta)}\left(\frac{b}{a-c}\right)^{1-\varepsilon} I\left(\frac{b}{a+b-c}, \varepsilon, 1-\varepsilon\right) \\
& +\frac{\pi \csc (\varepsilon \pi)}{a(\delta-\theta)}\left(\frac{a}{b-c}\right)^{\varepsilon} I\left(\frac{a}{a+b-c}, 1-\varepsilon, \varepsilon\right),
\end{aligned}
$$

where $I(z, \rho, v)$ stands for the incomplete beta function ratio for $0 \leq z \leq 1, \rho>0$ and $v>0$, which is defined by

$$
I(z, \rho, v)=\frac{1}{B(\rho, v)} \int_{0}^{z} t^{\rho-1}(1-t)^{v-1} \mathrm{~d} t,
$$

here $B(\rho, v)$ is the beta function given by

$$
B(\rho, v)=\int_{0}^{1} t^{\rho-1}(1-t)^{v-1} \mathrm{~d} t .
$$

Lemma 5 [23]. For system (4), assume $V(z): R^{n} \rightarrow R$ is a C-regular function. If there exist constants $a>0, \quad b>0,0<\beta<2 \sqrt{a b}, \delta>1$ and $0<\theta<1$ satisfying $\delta+\theta=2$ such that

$$
£ V(z) \leq \beta V(z)-a V^{\theta}(z)-b V^{\delta}(z), \quad z \in R^{n} \backslash\{0\},
$$

the zero solution of system (4) is FXT stable and its ST can be estimated by 


$$
E\left(T\left(z_{0}, \omega\right)\right) \leq T_{\max }^{5}=\frac{1}{\delta-1} \frac{2}{\sqrt{4 a b-\beta^{2}}}\left(\frac{\pi}{2}+\arctan \left(\frac{\beta}{\sqrt{4 a b-\beta^{2}}}\right)\right) .
$$

Lemma 6 [24]. For system (4), assume that $V(z): R^{n} \rightarrow R^{+} \cup\{0\}$ is a C-regular function. If there exist constants $\gamma, a, b>0,0<\theta<1$ and $\delta>1$ satisfying $\theta+\delta=2$ such that

$$
£ V(z) \leq-\gamma V(z)-a V^{\theta}(z)-b V^{\delta}(z), \quad z \in R^{n} \backslash\{0\} .
$$

Then the zero solution of system (4) is FXT stable and its corresponding ST can be estimated as $E\left(T\left(z_{0}, \omega\right)\right) \leq T_{\max }^{6}$, where

$$
T_{\max }^{6} \triangleq \begin{cases}\frac{2}{(\delta-1) \sqrt{\Delta}}\left(\frac{\pi}{2}-\arctan \frac{\gamma}{\sqrt{\Delta}}\right), & 0<\gamma<2 \sqrt{a b}, \\ \frac{2}{\gamma(\delta-1)}, & \gamma=2 \sqrt{a b}, \\ \frac{1}{(\delta-1) \sqrt{-\Delta}} \ln \frac{\gamma+\sqrt{-\Delta}}{\gamma-\sqrt{-\Delta}}, & \gamma>2 \sqrt{a b},\end{cases}
$$

here $\Delta=4 a b-\gamma^{2}$.

Lemma 7 [21]. If $v_{1}, v_{2}, \cdots, v_{m} \geq 0,0<\eta \leq 1, \kappa>1$, then

$$
\sum_{s=1}^{m} v_{s}^{\eta} \geq\left(\sum_{s=1}^{m} v_{s}\right)^{\eta}, \quad \sum_{s=1}^{m} v_{s}^{\kappa} \geq m^{1-\kappa}\left(\sum_{s=1}^{m} v_{s}\right)^{\kappa} .
$$

Lemma 8 [22]. Let $v$ and $z$ be any two column vectors in $R^{m}$, then the following matrix inequality is satisfied for any positive definite matrix $Q \in R^{m \times m}$.

$$
2 v^{\mathrm{T}} z \leq v^{\mathrm{T}} Q v+z^{\mathrm{T}} Q^{-1} z \text {. }
$$

\section{Main Results}

In this section, based on the FXT stability results introduced in above section, we will derive some sufficient criteria for the FXT synchronization between the drive-response systems (1) and (2). To this, first we design the controller $u(t)$ in response system (2) as follows:

$$
u(t)=-\Lambda e(t)-\rho e^{[p]}(t)-\xi e^{[q]}(t),
$$

where $\Lambda=\operatorname{diag}\left(\lambda_{1}, \lambda_{2}, \cdots, \lambda_{n}\right)$ is a positive constant matrix. $\rho$ and $\xi$ are the tunable constants, and $p$ and $q$ are the real numbers such that

$$
\begin{gathered}
0<p<1, q>1 .|e(t)|^{s}=\left(\left|e_{1}(t)\right|^{s},\left|e_{2}(t)\right|^{s}, \cdots,\left|e_{n}(t)\right|^{s}\right)^{\mathrm{T}}, \\
\operatorname{sgn}(e(t))=\operatorname{diag}\left(\operatorname{sgn}\left(e_{1}(t)\right), \operatorname{sgn}\left(e_{2}(t)\right), \cdots, \operatorname{sgn}\left(e_{n}(t)\right)\right), \text { and } \\
e^{[s]}(t) \cong \operatorname{sgn}(e(t))|e(t)|^{s} .
\end{gathered}
$$

Then, under controller (10), the error system (3) can be rewritten as follows:

$$
\begin{aligned}
\mathrm{d} e(t)= & {\left[-D e(t)+A g(e(t))-\Lambda e(t)-\rho \mathrm{e}^{[p]}(t)-\xi \mathrm{e}^{[q]}(t)\right] \mathrm{d} t } \\
& +\sigma(t, e(t)) \mathrm{d} \omega(t) .
\end{aligned}
$$

Now let $L=\operatorname{diag}\left(L_{1}, L_{2}, \cdots, L_{n}\right)$, then based on the FXT controller (12), the 
following results can be derived.

Theorem 1. Suppose that the Assumptions 1 and 2 are satisfied, if the control gain matrix $\Lambda$ satisfy the following matrix inequality

$$
\Pi \triangleq\left[\begin{array}{cc}
2(D+\Lambda)-Q_{1}-G & L A^{\mathrm{T}} \\
A L & Q_{1}
\end{array}\right]>0,
$$

where $Q_{1}$ is an arbitrary $n \times n$ positive matrix. Then the drive-response networks (1) and (2) can be FXT synchronized in probability via controller (10), and its corresponding ST can be estimated by

$$
E\left(T\left(e_{0}, \omega\right)\right) \leq T_{\max }^{7}=\min \left\{T_{\max }^{2}, T_{\max }^{3}\right\},
$$

where $T_{\max }^{2}$ and $T_{\max }^{3}$ are respectively given in Lemma 3 and Lemma 4 with the parameters $c=-\lambda_{\max }\left(-2(D+\Lambda)+Q_{1}+L A^{\mathrm{T}} Q_{1}^{-1} A L+G\right), \quad a=2 \rho$, $\theta=\frac{1+p}{2}, b=2 n^{\frac{1-q}{2}} \xi$ and $\delta=\frac{1+q}{2}$.

Proof. First, we construct the following Lyapunov function

$$
V(t)=e^{\mathrm{T}}(t) e(t) .
$$

Then, by calculating the $£ V(t)$ along the trajectories of error system (3), we get

$$
\begin{aligned}
£ V(t)= & 2 e^{\mathrm{T}}(t)\left[-D e(t)+A g(e(t))-\Lambda e(t)-\rho \mathrm{e}^{[p]}(t)-\xi \mathrm{e}^{[q]}(t)\right] \mathrm{d} t \\
& +\operatorname{trace}\left[\sigma^{\mathrm{T}}(t, e(t)) \sigma(t, e(t))\right] .
\end{aligned}
$$

By Lemma 8 and Assumption 1, we obtain the following inequality:

$$
\begin{aligned}
2 e^{\mathrm{T}}(t) A g(e(t)) & \leq e^{\mathrm{T}}(t) Q_{1} e(t)+g^{\mathrm{T}}(e(t)) A^{\mathrm{T}} Q_{1}^{-1} A g(e(t)) \\
& \leq e^{\mathrm{T}}(t) Q_{1} e(t)+e^{\mathrm{T}}(t) L A^{\mathrm{T}} Q_{1}^{-1} A L e(t) .
\end{aligned}
$$

By Assumption 2, we have

$$
\text { trace }\left[\sigma^{\mathrm{T}}(t, e(t)) \sigma(t, e(t))\right] \leq e^{\mathrm{T}}(t) G e(t) .
$$

Also, it is not difficult to check that

$$
e^{\mathrm{T}}(t) e^{[p]}(t)=e^{\mathrm{T}}(t) \operatorname{sgn}(e(t))|e(t)|^{p}=\sum_{i=1}^{n}\left|e_{i}(t)\right|^{1+p}
$$

and

$$
e^{\mathrm{T}}(t) e^{[q]}(t)=e^{\mathrm{T}}(t) \operatorname{sgn}(e(t))|e(t)|^{q}=\sum_{i=1}^{n}\left|e_{i}(t)\right|^{1+q} .
$$

Let $\Upsilon \triangleq-2(D+\Lambda)+Q_{1}+L A^{\mathrm{T}} Q_{1}^{-1} A L+G$, and using the well-known Schur complement equivalence [32] to $\Pi>0$, which is defined in (15), we obtain $-\Upsilon>0$ or $\Upsilon<0$. Thus, by substituting (16), (17), (18) and (19) to (15), we have

$$
\begin{aligned}
£ V(t) \leq & e^{\mathrm{T}}(t)\left[-2(D+\Lambda)+Q_{1}+L A^{\mathrm{T}} Q_{1}^{-1} A L+G\right] e(t) \\
& -2 \rho e^{\mathrm{T}}(t) e^{[p]}(t)-2 \xi e^{\mathrm{T}}(t) e^{[q]}(t) \\
\leq & \lambda_{\max }(\Upsilon) e^{\mathrm{T}}(t) e(t)-2 \rho \sum_{i=1}^{n}\left|e_{i}(t)\right|^{1+p}-2 \xi \sum_{i=1}^{n}\left|e_{i}(t)\right|^{1+q} .
\end{aligned}
$$

By Lemma 7, we can obtain that 


$$
\sum_{i=1}^{n}\left|e_{i}(t)\right|^{1+p} \geq\left(\sum_{i=1}^{n}\left|e_{i}(t)\right|^{2}\right)^{\frac{1+p}{2}}=V^{\frac{1+p}{2}}(t)
$$

and

$$
\sum_{i=1}^{n}\left(e_{i}^{2}(t)\right)^{\frac{1+q}{2}} \geq n^{\frac{1-q}{2}}\left(\sum_{i=1}^{n} e_{i}^{2}(t)\right)^{\frac{1+q}{2}}=n^{\frac{1-q}{2}} V^{\frac{1+q}{2}}(t)
$$

In view of (22), (21) and (22), we can have

$$
\begin{aligned}
£ V(t) & \leq \lambda_{\max }(\Upsilon) V(t)-2 \rho V^{\frac{1+p}{2}}(t)-2 n^{\frac{1-q}{2}} \xi V^{\frac{1+q}{2}}(t) \\
& =\lambda_{\max }(\Upsilon) V(t)-a V^{\theta}(t)-b V^{\delta}(t),
\end{aligned}
$$

where $a=2 \rho, \theta=\frac{1+p}{2}, b=2 n^{\frac{1-q}{2}} \xi$ and $\delta=\frac{1+q}{2}$.

Therefore, we can conclude from the Lemmas 3 and 4 that the origin of error system (3) is FXT stable in probability and its ST can be estimated by

$$
E\left(T\left(e_{0}, \omega\right)\right) \leq T_{\max }^{7}=\min \left\{T_{\max }^{2}, T_{\max }^{3}\right\},
$$

where $T_{\max }^{2}$ and $T_{\max }^{3}$ are given in Lemmas 3 and 4 respectively, and their parameters are chosen as $c=-\lambda_{\max }(\Upsilon), \quad a=2 \rho, \quad \theta=\frac{1+p}{2}, \quad b=2 n^{\frac{1-q}{2}} \xi$, $\delta=\frac{1+q}{2}$. The proof is achieved.

When $p+q=2$ in the controller (10), we have a following Corollary from Theorem 1 and Lemma 6.

Corollary 1. Suppose that $p+q=2$ in the controller (10), if Assumption 1, Assumption 2 and matrix inequality (15) are satisfied, then the drive-response networks (1) and (2) can be FXT synchronized in probability under controller (10), and its $S T$ is estimated by $E\left(T\left(e_{0}, \omega\right)\right) \leq T_{\max }^{6}$, where $T_{\max }^{6}$ is given in Lemma 6 with the parameters $\gamma=-\lambda_{\max }(\Upsilon)>0, \quad a=2 \rho, \quad \theta=\frac{1+p}{2}$, $b=2 n^{\frac{1-q}{2}} \xi$ and $\delta=\frac{1+q}{2}$.

Proof. Similar to proof of Theorem 1, we know that the inequality (24) is satisfied under the conditions of Corollary 1. Thus from Lemma 6, we can obtain that the conclusions of Corollary 1 hold true. The proof is completed.

In the following, we will realize the fixed time synchronization between the systems (1) and (2) via designing a simplified controller given as follows

$$
u(t)=-\rho e^{[p]}(t)-\xi e^{[q]}(t)
$$

where parameters $\rho, \xi, p$ and $q$ are the same as defined in controller (10).

Theorem 2. Suppose that $Q_{2}$ is an arbitrary $n \times n$ positive matrix and the Assumptions 1 and 2 hold true, then the drive-response systems (1) and (2) will realize FXT synchronization in probability via controller (24). Moreover, its corresponding $S T$ can be estimated as $E\left(T\left(e_{0}, \omega\right)\right) \leq T_{\max }^{8}$, where 


$$
T_{\max }^{8} \triangleq \begin{cases}T_{\max }^{4}, & 0<\tilde{c}<\min \{a, b\}, \\ T_{\max }^{3}, & \tilde{c}=0, \\ \min \left\{T_{\max }^{2}, T_{\max }^{3}\right\}, & \tilde{c}<0,\end{cases}
$$

here $\tilde{c}=\lambda_{\max }\left(-2 D+Q_{2}+L A^{\mathrm{T}} Q_{2}^{-1} A L+G\right)$, and the $T_{\max }^{2}, T_{\max }^{3}, T_{\max }^{4}$ are given in Lemmas 3 and 4, and their parameters are chosen as $c=-\tilde{c}, a=2 \rho$, $\theta=\frac{1+p}{2}, \quad b=2 n^{\frac{1-q}{2}} \xi$ and $\delta=\frac{1+q}{2}$.

Proof. We again chose the Lyapunov function as $V(t)=e^{T}(t) e(t)$. Then, under controller (24), we have

$$
\begin{aligned}
£ V(t)= & 2 e^{\mathrm{T}}(t)\left[-D e(t)+A g(e(t))-\rho \operatorname{sgn}(e(t))|e(t)|^{p}\right. \\
& \left.-\xi \operatorname{sgn}(e(t))|e(t)|^{q}\right]+\operatorname{trace}\left[\sigma^{\mathrm{T}}(t, e(t)) \sigma(t, e(t))\right] .
\end{aligned}
$$

By Equations (16)-(19), we have

$$
\begin{aligned}
£ V(t) \leq & e^{\mathrm{T}}(t)\left[-2 D+Q_{2}+L A^{\mathrm{T}} Q_{2}^{-1} A L+G\right] e(t) \\
& -2 \rho\left(|e(t)|^{p}\right)^{\mathrm{T}}|e(t)|-2 \xi\left(|e(t)|^{q}\right)^{\mathrm{T}}|e(t)| \\
\leq & \lambda_{\max }\left[-2 D+Q_{2}+L A^{\mathrm{T}} Q_{2}^{-1} A L+G\right] e^{\mathrm{T}}(t) e(t) \\
& -2 \rho\left(|e(t)|^{p}\right)^{\mathrm{T}}|e(t)|-2 \xi\left(|e(t)|^{q}\right)^{\mathrm{T}}|e(t)| \\
\leq & \tilde{c} e^{\mathrm{T}}(t) e(t)-2 \rho\left(|e(t)|^{p}\right)^{\mathrm{T}}|e(t)|-2 \xi\left(|e(t)|^{q}\right)^{\mathrm{T}}|e(t)| \\
\leq & \tilde{c} V(t)-2 \rho\left(|e(t)|^{p}\right)^{\mathrm{T}}|e(t)|-2 \xi\left(|e(t)|^{q}\right)^{\mathrm{T}}|e(t)| .
\end{aligned}
$$

Introducing (21) and (22) to (26) yields

$$
\begin{aligned}
£ V(t) & \leq \tilde{c} V(t)-2 \rho V^{\frac{1+p}{2}}(t)-2 n^{\frac{1-q}{2}} \xi V^{\frac{1+q}{2}}(t) \\
& =\tilde{c} V(t)-a V^{\theta}(t)-b V^{\delta}(t) .
\end{aligned}
$$

According to Lemma 4, the drive-response networks (1) and (2) will achieve FXT synchronization in probability. In addition, its ST $T_{\max }^{8}$ can estimate through following analysis.

1) If $0<\tilde{c}<\min \{a, b\}$, then from Lemma 4 , we can get that $T_{\max }^{8}=T_{\max }^{4}$.

2) If $\tilde{c}=0$, then from Lemma 4 again, we can have that $T_{\max }^{8}=T_{\max }^{3}$.

3) If $\tilde{c}<0$, then from Lemmas 3 and 4 , we can obtain that $T_{\max }^{8}=\min \left\{T_{\max }^{2}, T_{\max }^{3}\right\}$.

where the parameters of $T_{\max }^{2}, T_{\max }^{3}$ and $T_{\max }^{4}$ are chosen as $\tilde{c}=\lambda_{\max }\left(-2 D+Q_{2}+L A^{\mathrm{T}} Q_{2}^{-1} A L+G\right), \quad a=2 \rho, \quad \theta=\frac{1+p}{2}, \quad b=2 n^{\frac{1-q}{2}} \xi$ and $\delta=\frac{1+q}{2}$.

The proof of Theorem 2 is completed.

When $p+q=2$ in the controller (24), we have a following result from Theorem 2 and Lemma 4. 
Corollary 2. Suppose that $p+q=2$ in controller (10) and the Assumptions 1 and 2 are satisfied, then the drive-response systems (1) and (2) will achieve FXT synchronization in probability via controller (24). Moreover, its corresponding $S T$ can be estimated as $E\left(T\left(e_{0}, \omega\right)\right) \leq T_{\max }^{9}$, where

$$
T_{\max }^{9} \triangleq \begin{cases}T_{\max }^{5}, & 0<\tilde{c}<2 \sqrt{a b}, \\ T_{\max }^{3}, & \tilde{c}=0, \\ T_{\max }^{6}, & \tilde{c}<0,\end{cases}
$$

here $\tilde{c}=\lambda_{\max }\left(-2 D+Q_{2}+L A^{\mathrm{T}} Q_{2}^{-1} A L+G\right), \quad T_{\max }^{3}, T_{\max }^{5}$ and $T_{\max }^{6}$ are respectively given in Lemmas 4,5 and 6 , and their parameters are chosen as $c=\gamma=-\tilde{c}=-\tilde{c}, \quad \beta=\tilde{c}, \quad a=2 \rho, \theta=\frac{1+p}{2}, b=2 n^{\frac{1-q}{2}} \xi$ and $\delta=\frac{1+q}{2}$.

Proof. From the proof of Theorem 2, we know that the inequality (28) is satisfied. Thus according to the Lemma 4, the drive-response networks (1) and (2) can be FXT synchronized in probability. In addition, its ST $T_{\max }^{9}$ can estimate through following analysis.

1) If $0<\tilde{c}<2 \sqrt{a b}$, then from Lemma 5 , we can get that $T_{\max }^{9}=T_{\max }^{5}$.

2) If $\tilde{c}=0$, then from Lemma 4 again, we can have that $T_{\max }^{9}=T_{\max }^{3}$.

3) If $\tilde{c}<0$, then from Lemma 6, we can obtain that $T_{\max }^{9}=T_{\max }^{6}$.

Where the parameters of $T_{\max }^{3}, T_{\max }^{5}$ and $T_{\max }^{6}$ are chosen as $c=-\tilde{c}, \beta=\tilde{c}$, $\gamma=-\tilde{c}, \quad a=2 \rho, \theta=\frac{1+p}{2}, \quad b=2 n^{\frac{1-q}{2}} \xi$ and $\delta=\frac{1+q}{2}$. The proof is completed.

Remark 1. As known to all, when study the synchronization issue of nonlinear systems, it is an important task to design a controller, and it is desirable to design the controller as simple as possible in order to save the control cost. In early published works [21] [23] [31] [33], however, the authors realized FXT synchronization of NNs via employing a type of hybrid controller $u_{i}(t)$ which composed of one linear term $-\lambda_{i} e_{i}(t)$ and two nonlinear terms $-\rho e^{[p]}(t)-\xi e^{[q]}(t)$. However, as we done in Theorem 2 and Corollary 2, when the linear term $-\lambda_{i} e_{i}(t)$ of $u_{i}(t)$ removed, the other two terms $-\rho e^{[p]}(t)-\xi e^{[q]}(t)$ still insures the FXT synchronization of considered networks. Thus the results of Theorem 2 and Corollary 2 are simpler and have a better applicability.

Remark 2. Similar to the most of the published works on FXT stabilization and synchronization, in Theorem 1 and Corollary 1, we have achieved to FXT synchronization by designing a types of controller $u_{i}(t)$ which composed of one linear term $-\lambda_{i} e_{i}(t)$ and two nonlinear terms $-\rho e^{[p]}(t)-\xi e^{[q]}(t)$. However, it is worth to note that, all of the three tunable parameters $\lambda_{i}, \rho$ and $\xi$ take effect in the estimation of the upper-bound of ST, and this can be seen the main advantages of Theorem 1 and Corollary 1 compared with the early published results [23] [31] [34] [35] [36].

Remark 3. As mentioned above, it is better to realize the FXT synchronization via using simple controller $u_{i}(t)=-\rho e^{[p]}(t)-\xi e^{[q]}(t)$. However, it is not diffi- 
cult to see that compared the results of Theorem 2 and Corollary 2, the results of Theorem 1 and Corollary 1 gives smaller estimation when the control gains $\lambda_{i}>0$ in controller $u_{i}(t)=-\lambda_{i} e_{i}(t)-\rho e^{[p]}(t)-\xi e^{[q]}(t)$, and the bigger $\lambda_{i}$ results in a smaller ST estimation. Therefore, the linear term $-\lambda_{i} e_{i}(t)$ should be added in accordance with the convergence time $T$ to be short and the control cost not to be high, considering the designer requirements.

\section{Numerical Examples and Simulations}

In this section, the following two numerical examples are provided to illustrate the effectiveness of the established theoretical results in above sections.

Example 1. For $\mathrm{n}=3$, consider the FXT synchronization between drive-response systems (1) and (2) with the following system parameters: $h(v)=\tanh (v), \quad J=\operatorname{diag}(0,0,0), \quad \sigma(t, x(t))=0.5 \operatorname{diag}\left(x_{1}(t), x_{2}(t), x_{3}(t)\right)$ and

$$
D=\left(\begin{array}{ccc}
0.94 & 0 & 0 \\
0 & 0.94 & 0 \\
0 & 0 & 0.94
\end{array}\right), \quad A=\left(\begin{array}{ccc}
1.65 & -4.224 & -4.224 \\
-4.224 & 1.452 & -5.808 \\
-4.224 & 5.808 & 1.32
\end{array}\right)
$$

Set the initial values of system (1) in Example 1 as $x_{1}(0)=-0.1, x_{2}(0)=0.2$ and $x_{3}(0)=0.1$, then the numerical simulation of system (1) with above parameters are illustrated in Figure 1, which shows that it has a chaotic attractor.

It is not difficult check that the Assumptions 1 and 2 are satisfied with $L=\operatorname{diag}(1,1,1)$ and $G=0.5 \operatorname{diag}(1,1,1)$. By using the LMI Toolbox in Matlab, the following solution are obtained for matrix inequality (15)

$$
Q_{1}=\left(\begin{array}{ccc}
16.4470 & 8.1872 & -6.3667 \\
8.1872 & 15.4389 & -0.0097 \\
-6.3667 & -0.0097 & 17.7886
\end{array}\right), \quad \Lambda=\left(\begin{array}{ccc}
14.4615 & 0 & 0 \\
0 & 14.4615 & 0 \\
0 & 0 & 14.4615
\end{array}\right) \text {. }
$$

Thus inequality (15) is also satisfied and $c=3.3655$. Now choosing two different set of parameters as follows: (i) $\rho=1.2, \xi=2.1, p=0.4$ and $q=1.8$; (ii) $\rho=1.2, \xi=2.1, p=0.2$ and $q=1.8$. Then, from Theorem 1 and Corollary 1 , the derive system (1) is FXT stochastic synchronized to response system (2) under the controller (10). The time evolution of synchronization errors between systems (1) and (2) for above two different set of parameters are shown in Figure 2 and Figure 3, respectively, where the initial conditions of response systems (2) are randomly chosen in $[-5,5]$. For case (i), from Theorem 1, by simple calculations we get $T_{\max }^{2}=1.4683, T_{\max }^{3}=1.7009$. Thus $T_{\max }^{7}=\min \left\{T_{\max }^{2}, T_{\max }^{3}\right\}=1.7009$. For case (ii), since $p+q=2$, we can get from Corollary 1 that $T_{\max }^{6}=1.1097$, while Lemma 2 and Lemma 3 give the ST estimations $T_{\max }^{2}=1.2513$ and $T_{\max }^{3}=1.4510$, respectively.

Example 2. For $n=3$, consider the FXT synchronization between drive-response systems (1) and (2) under the controller (24) with the following system parameters: $h(v)=\tanh (v), J=\operatorname{diag}(0,0,0)$, $\sigma(t, x(t))=0.5 \operatorname{diag}\left(x_{1}(t), x_{2}(t), x_{3}(t)\right)$ and 

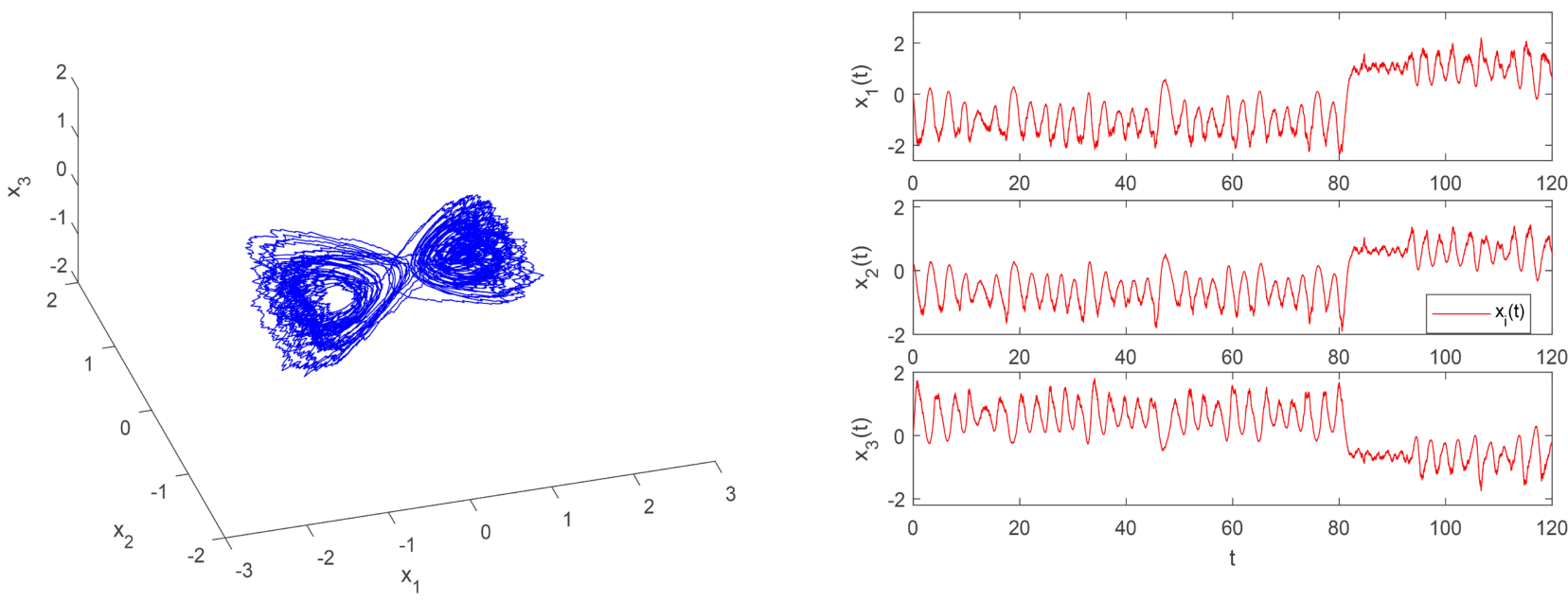

Figure 1. The transient behavior of system (1) in Example 1.

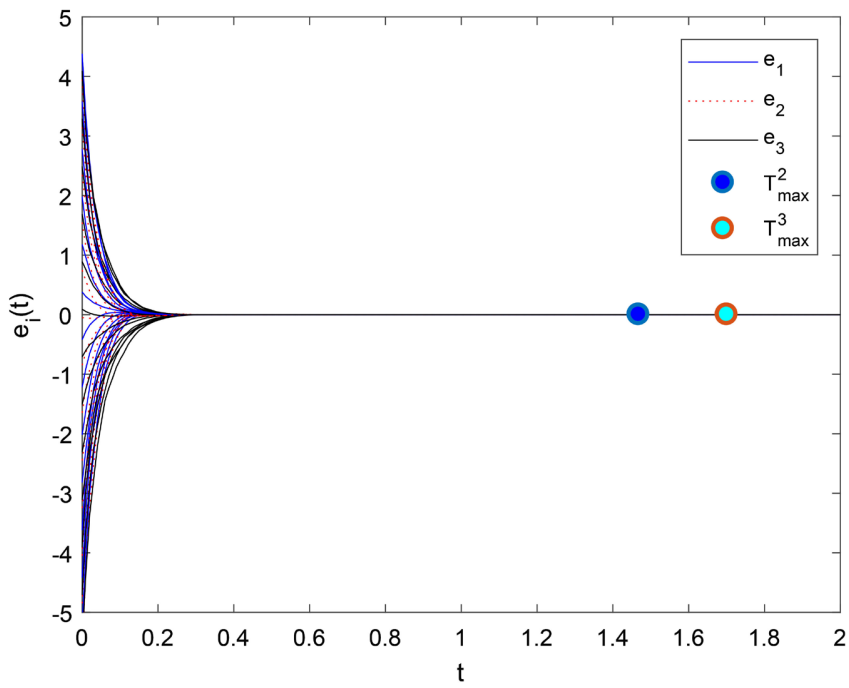

Figure 2. Evaluation of synchronization errors for case (i).

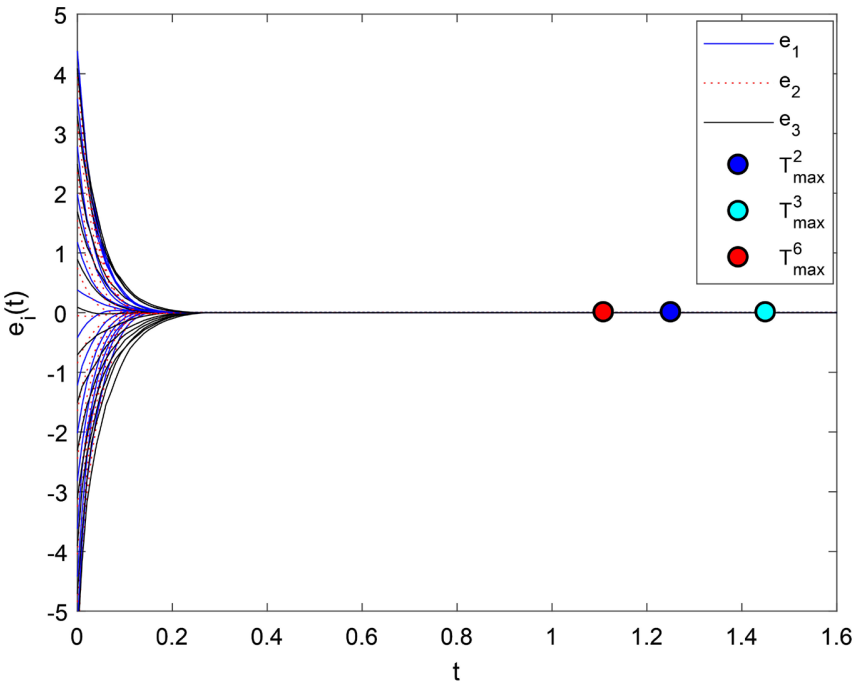

Figure 3. Evaluation of synchronization errors for case (ii). 


$$
D=\left(\begin{array}{ccc}
0.94 & 0 & 0 \\
0 & 0.94 & 0 \\
0 & 0 & 0.94
\end{array}\right), \quad A=0.5\left(\begin{array}{ccc}
0.825 & -2.112 & -2.112 \\
-2.112 & 0.726 & -2.904 \\
-2.112 & 2.9048 & 0.66
\end{array}\right)
$$

Now set the initial values of system (1) in Example 2 as $x_{1}(0)=-0.6087$, $x_{2}(0)=-0.2560$ and $x_{3}(0)=0.4875$, then the numerical simulation of system (1) with above parameters are illustrated in Figure 4, which shows that it also has a chaotic attractor.

It is not difficult check that $L=\operatorname{diag}(1,1,1), G=0.5 \operatorname{diag}(1,1,1)$. Letting $Q_{2}=4.5 * \operatorname{diag}(1,1,1)$, then by simple calculation we can get that $\tilde{c}=\lambda_{\max }\left(-2 D+Q_{2}+L A^{\mathrm{T}} Q_{2}^{-1} A L+G\right)=7.8766$. Now choosing two different set of parameters such that (i) $\rho=8, \xi=8.1, p=0.4$ and $q=1.8$; (ii) $\rho=8$, $\xi=8.1, p=0.4$ and $q=1.6$. Then, all the conditions of Theorem 2 and Corollary 2 are satisfied for case (i) and case (ii). Therefore, from Theorem 2 and Corollary 2, the derive system (1) is FXT stochastic synchronized to response system (2) under the controller (24). The time evolution of synchronization errors between systems (1) and (2) for above two different set of parameters are shown in Figure 5 and Figure 6 respectively, where the initial conditions of response systems (2) are randomly chosen in $[-6,6]$. For case (i), since $\tilde{c}>0$, we can calculate from Theorem 2 that $T_{\max }^{4}=0.7796$. Thus

$T_{\max }^{8}=T_{\max }^{4}=0.7796$. For case (ii), since $p+q=2$ and $\tilde{c}>0$, we can get from Corollary 2 that $T_{\max }^{9}=T_{\max }^{5}=0.4751$, while Lemma 1 and gives the ST estimation $T_{\max }^{1}=1.3859$ for case (i) and $T_{\max }^{1}=1.2934$ for case (ii), respectively.

Remark 4. From the above two examples, we can see that the settling time estimations obtained through Theorems 1,2 and Corollaries 1, 2 are more accurate compared to the early published results [37] [38] [39] [40] [41]. From this point, results obtained in this paper are more general and have better applicability.
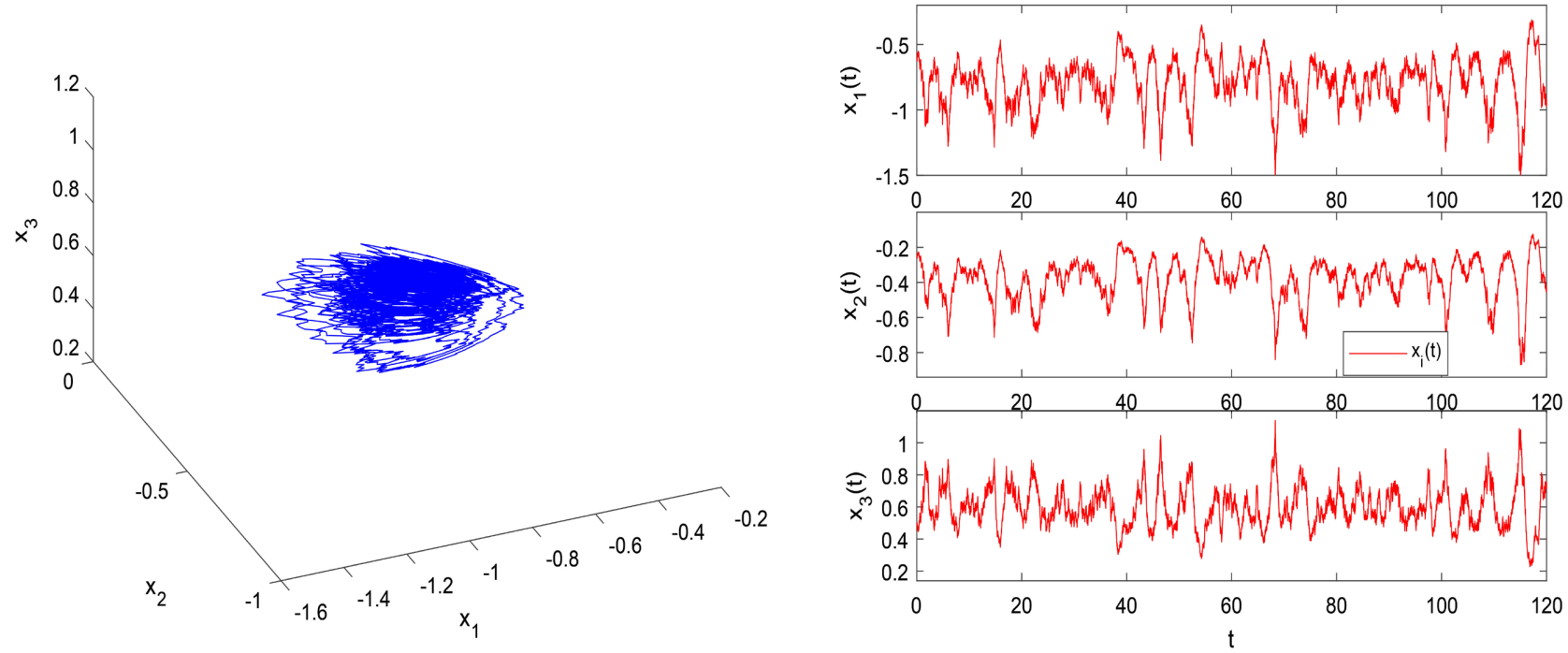

Figure 4. The transient behavior of system (1) in Example 2. 


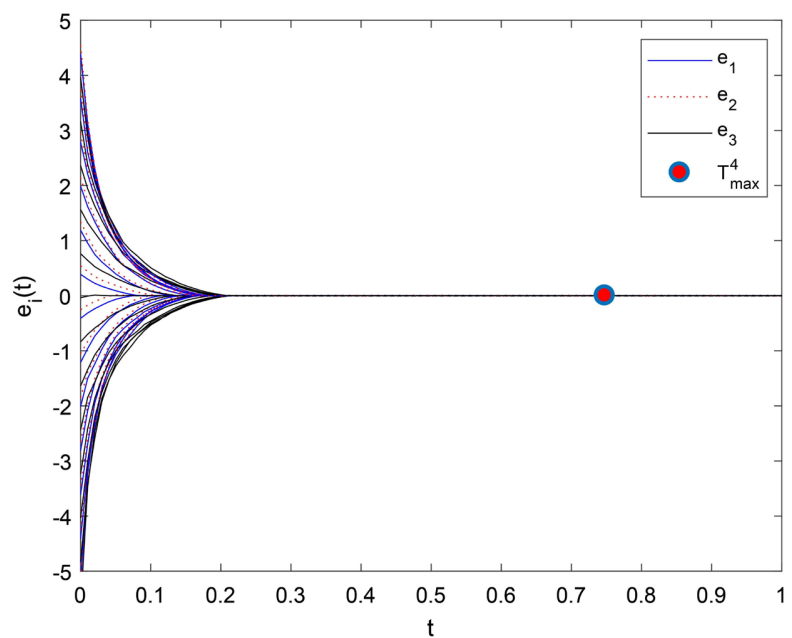

Figure 5. Evaluation of synchronization errors for case (i).

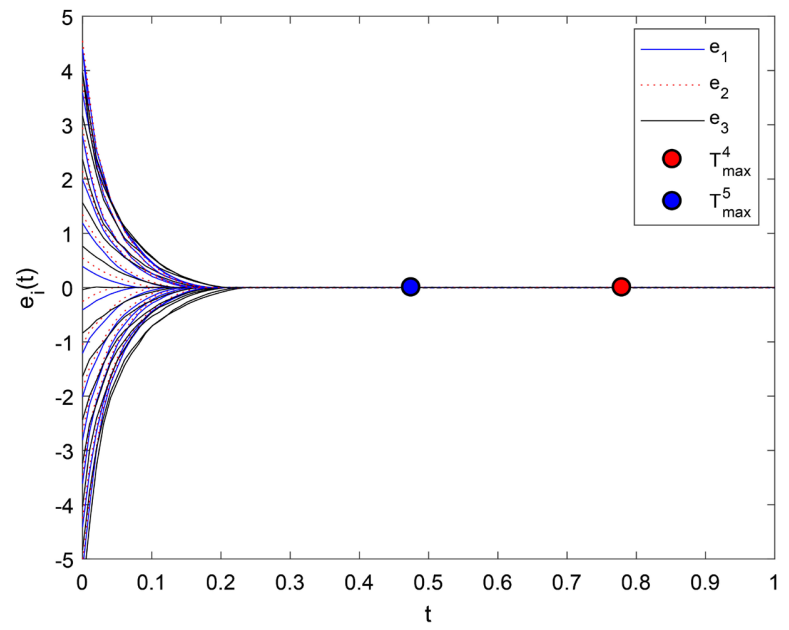

Figure 6. Evaluation of synchronization errors for case (ii).

\section{Conclusions}

In this paper, first, some recently developed new results on the FXT stability of deterministic dynamical systems are extended to stochastic dynamical systems. First, some earlier results on FXT stability of deterministic nonlinear systems are extended to the stochastic nonlinear systems. Then, based on these results, some simple sufficient conditions insuring the FXT synchronization of considered networks are derived by introducing two types of FXT controllers and utilizing some inequality techniques. Finally, our theoretical results are illustrated via giving two numerical examples with their Matlab simulations.

Recently, the FXT stability and synchronization of impulsive neural networks have been studied. However, there are very few works on the FXT synchronization issue of the stochastic neural networks with impulsive effects; this issue may be somewhat challenging since we have to deal with the effects of caused by impulsive term and stochastic perturbations at the same time, and it will be one of our future studying directions. 


\section{Funding}

This work was supported by the National Innovation Training Program for College Students (Grant no. 202010755075).

\section{Acknowledgements}

Not applicable.

\section{Conflicts of Interest}

The authors declare no conflicts of interest regarding the publication of this paper.

\section{References}

[1] Wan, Y., Cao, J., Wen, G. and Yu, W. (2016) Robust Fixed-Time Synchronization of Delayed Cohen-Grossberg Neural Networks. Neural Networks, 73, 86-94. https://doi.org/10.1016/j.neunet.2015.10.009

[2] Li, X.M., Huang, L.H. and Zhu, H. (2003) Global Stability of Cellular Neural Networks with Constant and Variable Delays. Nonlinear Analysis: Theory, Methods and Applications, 53, 319-333. https://doi.org/10.1016/S0362-546X(02)00176-1

[3] Zhou, Q. (2009) Global Exponential Stability of BAM Neural Networks with Distributed Delays and Impulses. Nonlinear Analysis. Real World Applications, 10, 144-153. https://doi.org/10.1016/j.nonrwa.2007.08.019

[4] Tsenov, G.T. and Mladenov, V.M. (2010) Speech Recognition Using Neural Networks. Proceedings of the 10 th Symposium on Neural Network Applications in Electrical Engineering (NEUREL), Belgrade, 23-25 September 2010, 181-186. https://doi.org/10.1109/NEUREL.2010.5644073

[5] Ouyang, D., Shao, J., Jiang, H., Nguang, S.K. and Shen, H.T. (2020) Impulsive Synchronization of Coupled Delayed Neural Networks with Actuator Saturation and Its Application to Image Encryption. Neural Networks, 128, 158-171.

https://doi.org/10.1016/j.neunet.2020.05.016

[6] Yang, X., Yang, Z. and Nie, X. (2014) Exponential Synchronization of Discontinuous Chaotic Systems via Delayed Impulsive Control and Its Application to Secure Communication. Communications in Nonlinear Science and Numerical Simulation, 19, 1529-1543. https://doi.org/10.1016/j.cnsns.2013.09.012

[7] Karakasoglu, A., Sudharsanan, S.I. and Sundareshan, M.K. (1993) Identification and Decentralized Adaptive Control Using Dynamical Neural Networks with Application to Robotic Manipulators. IEEE Transactions on Neural Networks, 4, 919-930. https://doi.org/10.1109/72.286887

[8] Zhang, C.K., He, Y., Jiang, L., Wang, Q.G. and Wu, M. (2017) Stability Analysis of Discrete-Time Neural Networks with Time-Varying Delay via an Extended Reciprocally Convex Matrix inequality. IEEE Transactions on Cybernetics, 47, 3040-3049. https://doi.org/10.1109/TCYB.2017.2665683

[9] Hu, C., Yu, J., Chen, Z., Jiang, H. and Huang, T. (2017) Fixed-Time Stability of Dynamical Systems and Fixed-Time Synchronization of Coupled Discontinuous Neural Networks. Neural Networks, 89, 74-83. https://doi.org/10.1016/j.neunet.2017.02.001

[10] Chen, T., Peng, S., Hong, Y. and Mai, G. (2020) Finite-Time Stability and Stabilization of Impulsive Stochastic Delayed Neural Networks with Rous and Rons. IEEE 
Access, 8, 87133-87141. https://doi.org/10.1109/ACCESS.2020.2992686

[11] Cai, Z. and Huang, L. (2018) Finite-Time Stabilization of Delayed Memristive Neural Networks: Discontinuous State-Feedback and Adaptive Control Approach. IEEE Transactions on Neural Networks and Learning Systems, 29, 856-868. https://doi.org/10.1109/TNNLS.2017.2651023

[12] Wang, L., Zeng, Z., Zong, X. and Ge, M.-F. (2019) Finite-Time Stabilization of Memristor-Based inertial Neural Networks with Discontinuous Activations and Distributed Delays. Journal of the Franklin Institute, 356, 3628-3643. https://doi.org/10.1016/j.jfranklin.2018.11.040

[13] Huang, X., Lin, W. and Yang, B. (2005) Global Finite-Time Stabilization of a Class of Uncertain Nonlinear Systems. Automatica, 41, 881-888. https://doi.org/10.1016/j.automatica.2004.11.036

[14] Lu, W., Liu, X. and Chen, T. (2016) A Note on Finite-Time and Fixed-Time Stability. Neural Networks, 8, 11-15. https://doi.org/10.1016/j.neunet.2016.04.011

[15] Polyakov, A. (2012) Nonlinear Feedback Design for Fixed-Time Stabilization of Linear Control Systems. IEEE Transactions on Automatic Control, 57, 2106-2110. https://doi.org/10.1109/TAC.2011.2179869

[16] Z. Zuo, Han, Q.-L., Ning, B., Ge, X. and Zhang, X.-M. (2018) An Overview of Recent Advances in Fixed-Time Cooperative Control of Multiagent Systems. IEEE Transactions on Industrial Informatics, 14, 2322-2334.

https://doi.org/10.1109/TII.2018.2817248

[17] Ni, J., Liu, L., Liu, C., Hu, X. and Li, S. (2017) Fast Fixed-Time Nonsingular Terminal Sliding Mode Control and Its Application to Chaos Suppression in Power System. IEEE Transactions on Circuits and Systems II: Express Briefs, 64, 151-155. https://doi.org/10.1109/TCSII.2016.2551539

[18] Zhang, W., Yang, S., Li, C. and Li, Z. (2019) Finite-Time and Fixed-Time Synchronization of Complex Networks with Discontinuous Nodes via Quantized Control. Neural Processing Letters, 50, 2073-2086. https://doi.org/10.1007/s11063-019-09985-9

[19] Giebel, S. and Rainer, M. (2011) Stochastic Processes Adapted By Neural Networks with Application to Climate, Energy, and Finance. Applied Mathematics and Computation, 218, 1003-1007. https://doi.org/10.1016/j.amc.2011.03.121

[20] Li, N., Wu, X., Feng, J. and Xu, Y. (2019) Fixed-Time Synchronization in Probability of Drive-Response Networks with Discontinuous Nodes and Noise Disturbances. Nonlinear Dynamics, 97, 297-311. https://doi.org/10.1007/s11071-019-04970-2

[21] Zhang, Y., Zhuang, J., Xia, Y., Bai, Y., Cao, J. and Gu, L. (2019) Fixed-Time Synchronization of the Impulsive Memristor-Based Neural Networks. Communications in Nonlinear Science and Numerical Simulation, 77, 40-53.

https://doi.org/10.1016/j.cnsns.2019.04.021

[22] Yang, X. and Cao, J. (2009) Stochastic Synchronization of Coupled Neural Networks with Intermittent Control. Physics Letters A, 373, 3259-3272.

https://doi.org/10.1016/j.physleta.2009.07.013

[23] Hu, C., He, H. and Jiang, H. (2020) Fixed/Preassigned-Time Synchronization of Complex Networks via Improving Fixed-Time Stability. IEEE Transactions on Cybernetics, 51, 2882-2892. https://doi.org/10.1109/TCYB.2020.2977934

[24] Abdurahman, A., Jiang, H. and Hu, C. (2021) Improved Fixed-Time Stability Results and Application to Synchronization of Discontinuous Neural Networks with State-Dependent Switching. International Journal of Robust and Nonlinear Control, 31, 5725-5744. https://doi.org/10.1002/rnc.5566 
[25] Yang, Y. and Cao, J. (2007) Exponential Lag Synchronization of a Class of Chaotic Delayed Neural Networks with Impulsive Effects. Physica A: Statistical Mechanics and Its Applications, 386, 492-502. https://doi.org/10.1016/j.physa.2007.07.049

[26] Liu, Y., Huang, J., Qin, Y. and Yang, X. (2020) Finite-Time Synchronization of Complex-Valued Neural Networks with Finite-Time Distributed Delays. Neurocomputing, 416, 152-157. https://doi.org/10.1016/j.neucom.2019.01.114

[27] Wei, R. and Cao, J. (2019) Fixed-Time Synchronization of Quaternion-Valued Memristive Neural Networks with Time Delays. Neural Networks, 113, 1-10. https://doi.org/10.1016/j.neunet.2019.01.014

[28] Li, X. and Song, S. (2014) Research on Synchronization of Chaotic Delayed Neural Networks with Stochastic Perturbation Using Impulsive Control Method. Communications in Nonlinear Science and Numerical Simulation, 19, 3892-3900. https://doi.org/10.1016/j.cnsns.2013.12.012

[29] Mao, X. (2008) Stochastic Differential Equations and Their Applications. 2nd Edition, Horwood Publishing, Chichester. https://doi.org/10.1533/9780857099402

[30] Yu, J., Yu, S., Li, J. and Yan, Y. (2018) Fixed-Time Stability Theorem of Stochastic Nonlinear Systems. International Journal of Control, 92, 2194-2200. https://doi.org/10.1080/00207179.2018.1430900

[31] Chen, C., Li, L., Peng, H., Yang, Y., Mi, L. and Zhao, H. (2020) A New Fixed-Time Stability Theorem and Its Application to the Fixed-Time Synchronization of Neural Networks. Neural Networks, 123, 412-419. https://doi.org/10.1016/j.neunet.2019.12.028

[32] Boyd, S., Ghaoui, L.E., Fernon, E. and Blakrishnan, V. (1994) Linear Matrix inequalities in System and Control Theory. Society for Industrial and Applied Mathematics, Philadelphia. https://doi.org/10.1137/1.9781611970777

[33] Hu, J. and Sui, G. (2019) Fixed-Time Control of Static Impulsive Neural Networks with infinite Distributed Delay and Uncertainty. Communications in Nonlinear Science and Numerical Simulation, 78, Article ID: 104848. https://doi.org/10.1016/j.cnsns.2019.05.006

[34] Alimi, A.M., Aouiti, C. and Assali E.A. (2019) Finite-Time and Fixed-Time Synchronization of a Class of inertial Neural Networks with Multi-Proportional Delays and Its Application to Secure Communication. Neurocomputing, 332, 29-43. https://doi.org/10.1016/j.neucom.2018.11.020

[35] Wei, R., Cao, J. and Alsaedi, A. (2018) Fixed-Time Synchronization of Memristive Cohen-Grossberg Neural Networks with Impulsive Effects. International Journal of Control, Automation and Systems, 16, 2214-2224. https://doi.org/10.1007/s12555-017-0788-5

[36] Zheng, M., Li, L., Peng, H., Xiao, J., Yang, Y. and Zhang, Y. (2018) Fixed-Time Synchronization of Memristive Fuzzy Bam Cellular Neural Networks with TimeVarying Delays Based on Feedback Controllers. IEEE Access, 6, 12085-12102. https://doi.org/10.1109/ACCESS.2018.2805183

[37] Li, J., Jiang, H., Hu, C. and Alsaedi, A. (2019) Finite/Fixed-Time Synchronization Control of Coupled Memristive Neural Networks. Journal of the Franklin Institute, 35, 9928-9952. https://doi.org/10.1016/j.jfranklin.2019.09.015

[38] Ao, W., Ma, T., Sanchez, R.-V. and Gan, H. (2020) Finite-Time and Fixed-Time Impulsive Synchronization of Chaotic Systems. Journal of the Franklin Institute, 357, 11545-11557. https://doi.org/10.1016/j.jfranklin.2019.07.023

[39] Li, R., Cao, J., Alsaedi, A. and Alsaadi, F. (2017) Exponential and Fixed-Time Syn- 
chronization of Cohen-Grossberg Neural Networks with Time-Varying Delays and Reaction-Diffusion Terms. Applied Mathematics and Computation, 313, 37-51. https://doi.org/10.1016/j.amc.2017.05.073

[40] Wang, S., Guo, Z., Wen, S., Huang, T. and Gong, S. (2020) Finite/Fixed-Time Synchronization of Delayed Memristive Reaction-Diffusion Neural Networks. Neurocomputing, 375, 1-8. https://doi.org/10.1016/j.neucom.2019.06.092

[41] Ji, G., Hu, C., Yu, J. and Jiang, H. (2018) Finite-Time and Fixed-Time Synchronization of Discontinuous Complex Networks: A Unified Control Framework Design. Journal of the Franklin Institute, 355, 4665-4685.

https://doi.org/10.1016/j.jfranklin.2018.04.026 